\title{
2 Medien im Leben von Kindern und Jugendlichen
}

Aktuelle Mediennutzungsstudien zeigen, dass der Stellenwert von Medien bereits bei Vorschulkindern groß ist, sich mit dem Älterwerden die Bandbreite der genutzten Medien weiter erhöht, und insgesamt die von Kindern und Jugendlichen mit Kommunikations- und Massenmedien verbrachte Zeit mit steigendem Alter zunehmend an Bedeutung gewinnt. Belegt werden soll dies nachfolgend anhand von Ergebnissen verschiedener Schülerbefragungen des Kriminologischen Forschungsinstituts Niedersachsen (KFN) sowie mit Daten regelmäßiger Befragungsstudien des Medienpädagogischen Forschungsverbundes Südwest (die so genannten KIM-Studien und JIM-Studien). Bezüglich des Fernsehens wird darüber hinaus auch auf Daten der AGF/GfK-Fernsehforschung zurückgegriffen. Dabei wird auch deutlich werden, dass neben dem Alter und dem Geschlecht der Kinder weitere soziodemografische und lebensweltliche Hintergrundfaktoren Verstärker oder Moderatoren der Rolle von Medien im kindlichen und jugendlichen Lebensalltag sind.

Mediennutzung ist Ausdruck kulturellen Handelns eines Individuums und von daher von Anfang an geprägt durch sein soziales und gesellschaftliches Umfeld, sie ist aber zugleich auch Ausdruck der Zugehörigkeit zu einer spezifischen Lebenswelt, einem spezifischen Milieu. Mit verschiedenen grundlegenden, und im Einzelnen sehr unterschiedlichen, theoretischen Konzepten von Bourdieu (1982) oder auch Schulze (1992) sowie lebensweltlichen Ansätzen aus dem Bereich der Marktforschung ${ }^{2}$ steht der Medienforschung inzwischen ein ganzes Bündel von Erklärungsmodellen individuellen Medienhandelns zur Verfügung, das auch von Seiten der Medienwirtschaft genutzt und weiterentwickelt ${ }^{3}$ wird. Auch einige Analysen der KIM-Studie 2006 basieren auf einem lebensweltlichen Erklärungsansatz (Feierabend \& Klingler, 2007b). Allen milieutheoretischen und lebensweltlichen Erklärungsansätzen von Freizeit und Medienhandeln ist dabei gemein, dass der Bildungshintergrund eines Individuums als zentraler milieu-

2 Den größten Bekanntheitsgrad dürften die Sinus-Milieus der Heidelberger Sinus Sociovision erreicht haben, die gerade als Analyseinstrumentarium in der Medien- und Mediaforschung große Verbreitung gefunden haben.

3 Etwa die ARD/ZDF-Mediennutzertypologie (Hartmann \& Höhne, 2007; Hartmann \& Neuwöhner, 1999), die einerseits zur Erklärung des Mediennutzungsverhaltens von Fernseh-, Radio-, und Internetnutzern verwendet wird (Oehmichen \& Schröter, 2008), andererseits aber auch als Instrument der Programm- und Mediaplanung verstanden wird. 
konstituierender Faktor gilt, der auch das Medienhandeln in entscheidender Weise prägt. Bezogen auf kindliche und jugendliche Mediennutzung bedeutet dies, dass sich Unterschiede der Mediennutzung nicht primär aufgrund des sozioökonomischen Status der Familie manifestieren sollten ${ }^{4}$, sondern vor allem mit der elterlichen Bildung und der Bildungsaffinität des Elternhauses sowie dem schulischen Bildungsumfeld der Kinder und Jugendlichen erklärt werden können.

\subsection{Vorschulkinder und Medien}

Zum Freizeit- und Medienverhalten zwei- bis fünfjähriger Vorschulkinder liegen mit einem Sonderschwerpunkt der Kinder- und Medien-Studie (KIM) 2003 relativ aktuelle Daten vor (Feierabend \& Mohr, 2004). Als Teil der KIM 2003 wurden 245 deutschlandweit repräsentativ ausgewählte Eltern von Vorschulkindern zum Medien- und Freizeitverhalten ihrer Kinder befragt. Die Autoren der Studie zeigen, dass hinter dem Spielen im Haus (72\%) die tägliche oder fast tägliche Fernsehnutzung bei 64 Prozent der zwei- fünfjährigen Kinder die zweithäufigste Tätigkeit der Kinder im Jahr 2003 war (S. 456). Zwei- bis Dreijährige schauten bereits zu 58 Prozent täglich oder fast täglich fern, Vier- bis Fünfjährige zu 69 Prozent. Darüber hinaus schauten sich 34 Prozent der Vorschulkinder täglich oder fast täglich Bilderbücher an (allein oder mit ihren Eltern), 30 Prozent hörten täglich oder fast täglich Radio. Neben diesen recht globalen Kennwerten zur täglichen beziehungsweise fast täglichen Verrichtung bestimmter Tätigkeiten wurden im Rahmen der KIM-Vorschulkinderstudie 2003 verschiedene mediale Freizeitaktivitäten der Kinder am Befragungsvortag mithilfe einer viertelstundengenauen Tätigkeitenliste abgefragt. Medientätigkeiten nahmen demnach 162 Minuten täglich ein, wobei Fernsehen, Video und DVD mit mehr als anderthalb Stunden (93 Minuten) täglicher Nutzungszeit das mit Abstand am häufigsten genutzte Medium darstellten (S. 458).

4 Was nicht bedeutet, das sozioökonomischer Status und Bildung nicht miteinander verknüpft sind, sondern nur, dass in der Konkurrenz beider Faktoren für die Erklärung medialer Nutzungsmuster der Bildung eine höhere Bedeutung zukommt. In den Analysen zur Mediengeräteausstattung und zu den Mediennutzungszeiten der befragten Viertklässler der KFN-Grundschulbefragung 2005 zeigte sich als durchgehender Befund, dass die Arbeitslosigkeit beider Eltern als ein zentrales Merkmal eines niedrigen sozioökonomischen Status für Mediengerätebesitz und Mediennutzungszeiten weniger Erklärungskraft besaß als die formale Bildung der Eltern (Mößle et al., 2007). 
Eine andere Datenquelle zur Erfassung der Fernsehzeiten von Klein- und Vorschulkindern stellt die Untersuchung von Vorschulkindern im AGF/GFKFernsehpanel dar, in dem Fernsehnutzungsdaten nicht in Fragebogenform, sondern technisch-verhaltensbasiert mithilfe eines aktiven telemetrischen Verfahrens erfasst werden (GfK-Fernsehforschung, 2005; Schwab \& Unz, 2004). Laut dieser Datenquelle verbrachten drei- bis fünfjährige Kinder im Jahr 2009 täglich durchschnittlich ${ }^{5} 71$ Minuten vor dem Fernseher (Feierabend \& Klingler, 2010, S. 183) $)^{6}$.

Vorschulkinder verfügen nach der KIM 2003 in einem gewissen Rahmen bereits über eine eigene Geräteausstattung im Kinderzimmer: Hatten unter zweibis dreijährigen Kindern im Jahr 2003 vier Prozent einen eigenen Fernseher im Zimmer stehen, waren es unter vier- bis fünfjährigen bereits zehn Prozent (Feierabend \& Mohr, 2004, S. 456). Dabei zeigen Feierabend und Klingler, dass ein eigener Fernseher im Zimmer die Fernsehzeiten von Klein- und Vorschulkindern deutlich erhöht. Drei- bis fünfjährige Kinder ohne ein eigenes Fernsehgerät schauten im Jahr 200964 Minuten täglich fern, Kinder dieser Altersgruppe mit eigenem Fernseher wiesen eine Sehdauer von 121 Minuten auf (Feierabend \& Klingler, 2010, S. 184 - 185). Laut Feierabend und Mohr (2004, S. 456) besaßen im Jahr 2003 rund sechs Prozent der zwei- bis dreijährigen Vorschulkinder beziehungsweise fünf Prozent der vier- bis fünfjährigen eine Spielkonsole, wobei innerhalb dieser Studie nicht erkennbar zwischen tragbaren (zum Beispiel Gameboy) und stationären Konsolen getrennt gefragt wurde. Interessanterweise zeigte sich bezüglich des Konsolenbesitzes bereits im Vorschulalter ein beachtlicher Geschlechterunterschied: Während Jungen und Mädchen dieser Altersgruppe im Jahr 2003 gleich viele Fernseher besaßen, verfügten doppelt so viele Jungen ( $8 \%$ ) wie Mädchen (4\%) über eine eigene Spielkonsole (S. 456). Einen eigenen Computer besaßen Zwei- bis Dreijährige im Jahr 2003 nicht, während die Eltern der vier- bis fünfjährigen Kinder in sechs Prozent der Fälle über

5 Wenn im Rahmen dieser Arbeit von Nutzungsdauer bzw. durchschnittlicher Tätigkeitsdauer gesprochen wird, sind - soweit nicht anders gekennzeichnet - in die Berechnung auch Personen einbezogen, welche die beschriebe Tätigkeit oder Mediennutzungsform gar nicht ausgeführt haben und insofern mit dem Wert 0 in die Berechnung eingehen. Bezogen auf die Fernsehnutzung entspricht das dem Begriff der Sehdauer.

6 Die Unterschiede zwischen den AGF/GFK-Daten und den Daten der KIM-Studie sind mit hoher Wahrscheinlichkeit den Unterschieden in der Untersuchungsmethode geschuldet und nicht Anzeichen eines veränderten Fernsehnutzungsverhaltens von Vorschulkindern zwischen 2003 (Erhebungszeitraum der KIM-Studie) und 2008 (GFK/AGFDaten). Für das Jahr 2003 weisen GFK/AGF eine Fernsehzeit von 69 Minuten aus (Feierabend \& Klingler, 2008). Nach dieser Datenquelle hat sich das Fernsehverhalten von Vorschulkindern also kaum geändert. 
eigene Computer im Kinderzimmer berichteten (ebenda). Auch hier gab es einen recht deutlichen Geschlechterunterschied, der aufgrund der geringen Fallzahlen allerdings nur schwer interpretierbar ist: Im Durchschnitt aller untersuchten Vorschulkinder verfügten deutlich mehr Mädchen (5\%) über einen eigenen Computer als Jungen (1\%) (ebenda).

Wenn auch über die Geräteausstattung und die mit Medien verbrachten Zeiten von Klein- und Vorschulkindern einige Daten vorliegen, so ist über die genutzten Medieninhalte nur wenig bekannt. Die differenziertesten Erkenntnisse gibt es noch zu inhaltlichen Fernsehvorlieben von Vorschulkindern, da die Fernsehnutzung aller Vorschulkinder ab drei Jahren, die in Haushalten des AGF/GFK-Fernsehpanels leben, einzeln erfasst wird. Mithilfe dieser Datenquelle lassen sich Aussagen über die Nutzungshäufigkeit verschiedener Fernsehprogramme und die Nutzung bestimmter Programmgenres machen. Nach Feierabend und Klingler (2010, S. 190) war der öffentlich-rechtliche Kinderkanal (KI.KA) im Jahr 2009 mit einem Marktanteil von 28,2 Prozent der beliebteste Fernsehsender unter drei- bis fünfjährigen Kindern. Der Privatsender Super-RTL lag mit 27,3 Prozent Marktanteil auf dem zweiten Platz, während alle anderen Sender mit einstelligen Marktanteilen in der Gruppe der Vorschulkinder nur eine recht geringe Rolle spielten. Unter den am häufigsten genutzten Fernsehgenres dominieren eindeutig fiktionale Formate mit 39 Prozent Anteil an der Gesamtfernsehzeit der Vorschulkinder (S. 193). Vorschulkinder sahen im Jahr 2009 darüber hinaus verhältnismäßig etwas mehr Fernsehwerbung (9\% der Gesamtfernsehzeit) als alle anderen Altersgruppen (7 bis $8 \%$ der Gesamtfernsehzeit) (ebenda). Unter den von den Kindern deutlich favorisierten fiktionalen Formaten dominierten eindeutig Zeichentrickfilme. Nach Daten des AGF/GFKFernsehpanels waren 70 Prozent der durch Vorschulkinder genutzten FictionFormate Animationen (S. 192).

\subsection{Kinder und Medien}

\subsubsection{Mediennutzungszeiten}

Die Befragung Kinder und Medien (KIM) 2008 des Medienpädagogischen Forschungsverbundes Südwest zeigt, dass Mediennutzung für Schulkinder im Alter von sechs bis dreizehn Jahren im Vergleich zu Vorschulkindern deutlich an Bedeutung gewinnt: In der für diese Altersgruppe deutschlandweit repräsentativen Befragung gaben 73 Prozent der befragten Kinder an, täglich oder fast täglich fernzusehen, womit das Fernsehen hinter dem Erledigen von 
Schulaufgaben (81 \%) auf der Rangliste der täglichen Freizeittätigkeiten auf dem zweiten Platz lag (Feierabend \& Rathgeb, 2009b, S. 9 - 10) ${ }^{7}$. Es folgten Spielen (draußen: $59 \%$; drinnen: $51 \%$ ), Treffen von Freunden (49\%), die Nutzung von Musikmedien (CD oder Kassette) und des Computers (beides $23 \%$ ) sowie des Radios (19\%) (ebenda). Das (fast) tägliche Lesen von Büchern lag dagegen mit einer Häufigkeit von 15 Prozent noch hinter der Nutzung tragbarer Spielkonsolen (16\%). Damit verliert die Zuwendung zu Büchern im Vergleich zu Kindern im Vorschulalter zunächst deutlich an Bedeutung. Von täglichen oder fast täglichen Unternehmungen mit der Familie berichteten 14 Prozent aller befragten Kinder (ebenda).

Die Ausnahmestellung des Fernsehens unter allen Freizeit- und Medienbeschäftigungen wird auch durch die starke Bindung der Kinder an das Fernsehen belegt: Danach gefragt, auf welches Medium die Kinder am wenigsten verzichten können, antworteten im Rahmen der KIM 200868 Prozent der Kinder, am wenigsten auf das Fernsehen verzichten zu können. Der Computer folgte mit 12 Prozent aller Nennungen erst mit weitem Abstand (Feierabend \& Rathgeb, 2009b, S. 56).

Auch in der KFN-Grundschulbefragung 2005, einer für neun westdeutsche Städte und Landkreise repräsentativen Befragung von 5.529 Schülerinnen und Schülern vierter Grundschulklassen, nahmen schulbezogene Tätigkeiten, Mediennutzung sowie Spielen mit Freunden und erwachsenen Familienmitgliedern den meisten Raum im Leben der befragten Kinder ein. Mithilfe einer auf den Tag vor dem Befragungstag bezogenen Tätigkeitenliste im Stil eines Freizeittagebuches war es - ähnlich wie bei der KIM-Vorschulkinderstudie 2003 - im Rahmen dieser Befragung möglich, das Verhalten der Kinder zeitlich recht genau zu umreißen: Rund sechs Stunden verbrachten die Schülerinnen und Schüler an einem durchschnittlichen Schultag im Frühjahr 2005 mit Schulbesuch und Hausaufgaben (364 Minuten), gut anderthalb Stunden beim Spiel mit Freundinnen oder Freunden (96 Minuten), anderthalb Stunden mit Fernsehen und Video ${ }^{8}$, eine gute Dreiviertelstunde ( 51 Minuten) bei Unternehmungen mit

7 Im Vergleich zur Vorgängerstudie KIM 2006 hat das Fernsehen allerdings leicht an Wichtigkeit verloren. Hier gaben noch 78 Prozent der Kinder an, täglich oder fast täglich fernzusehen (Feierabend \& Klingler, 2006).

8 Beim Vergleich zwischen Minutenangaben der KIM-Vorschulkinder-Studie und Daten der KFN-Grundschülerbefragung ist darauf zu achten, dass die KFN-Daten in der Regel auf Werktage (Schultage) bezogen sind und die KIM-Daten nicht zwischen Schul- und Wochentagen unterscheiden. Vor diesem Hintergrund ist der Befund zu erklären, dass Vorschulkinder der KIM-Studie anscheinend länger fern sehen (93 Minuten) als die Viertklässler der KFN-Studie (90 Minuten). Verrechnet man die auf Schultage bezogenen Fernsehnutzungszeiten der im Rahmen der KFN-Studie dienstags bis freitags befragten 
erwachsenen Freunden und Familienmitgliedern und mit dem Spielen von Video- und Computerspielen schließlich 29 Minuten. Das Lesen von Büchern oder Zeitschriften (wobei Lesen für die Schule ausdrücklich nicht angegeben werden sollte) hatte mit schultäglichen Nutzungszeiten von 50 Minuten einen mit Blick auf die niedrigen täglichen Nutzungshäufigkeiten im Rahmen der KIM-Studie - überraschend hohen Stellenwert (Mößle et al., 2007) ${ }^{9}$.

Bereits bei Grundschulkindern sind deutliche Unterschiede zwischen Jungen und Mädchen im Medienverhalten zu verzeichnen. Relativ gesehen präferierten Mädchen gegenüber den Jungen nach Daten der KIM-Studie 2008 die Rezeption von Musikkassetten oder -CDs), während die befragten Jungen zwischen sechsund dreizehn Jahren deutlich lieber Computer- und Videospiele spielten. Das Fernsehen war beiden Geschlechtern dagegen gleich wichtig: 32 Prozent der Mädchen und 30 Prozent der Jungen gaben das Fernsehen als eine von drei möglichen Lieblingsaktivitäten an (Feierabend \& Rathgeb, 2009b). Diese unterschiedlichen Präferenzen spiegeln sich auch in den Zeiten wieder, die Mädchen und Jungen mit verschiedenen Tätigkeiten verbringen. Die größten Unterschiede zeigen sich im Hinblick auf das Computer- und Videospielen. Nach Daten der KFN-Grundschulbefragung 2005 nutzten Jungen täglich 43 Minuten Computer-

Schülerinnen und Schüler mit den montags befragten Schulkindern, deren Angaben sich auf einen Wochenendtag beziehen (Fernsehen/Video/DVD: 133 Minuten), kommt man auf 102 Minuten tägliche Nutzung von Fernsehen/Video/DVD bei Grundschulkindern vierter Klassen.

9 Validieren lassen sich diese Angaben durch den Vergleich mit Daten der KIM-Studie 2008, in deren Rahmen Eltern nach den Mediennutzungszeiten ihrer Kinder gefragt wurden. Die Eltern berichteten hier von 91 Minuten Fernsehzeit der sechs- bis dreizehnjährigen Kinder, was gut mit den Daten der KFN-Schülerbefragung korrespondiert. Auch die Verhaltensdaten der AGF/GFK-Fernsehforschung weisen ähnliche Fernsehzeiten für diese Altersgruppe aus: Im Jahr 2009 schauten demnach sechs- bis neunjährige Kinder 80 Minuten fern, zehn- bis dreizehnjährige Kinder 100 Minuten (Feierabend \& Klingler, 2010). Insgesamt ergibt sich so eine relativ deutliche Übereinstimmung der Studien bezüglich der Fernsehnutzung von Kindern. Die Nutzung des Computers durch sechs bis dreizehnjährige Kinder gaben Eltern im Rahmen der KIM-Studie 2008 mit 40 Minuten an (Feierabend \& Rathgeb, 2009b), die Nutzung von Computer- und Konsolenspielen mit 29 Minuten Auch diese Angaben korrespondiert mit den in der KFNGrundschulbefragung 2005 erhobenen 29 Minuten Computer- und Konsolenspielzeit. Deutliche Abweichungen ergeben sich jedoch zwischen den Daten der KFNGrundschulbefragung 2005 und der KIM-Studie 2008 bezüglich des Lesens. Die Elternangaben im Rahmen der KIM-Studie weisen lediglich 23 Minuten tägliche Lesezeit aus (Feierabend \& Rathgeb, 2009b). Hier spielt mutmaßlich die Tatsache eine Rolle, dass im Rahmen der KFN-Befragung auch das Lesen von Comics und Zeitschriften explizit unter die Kategorie "Lesen" gefasst wurde (Mößle et al., 2007). Über alle durch mehrere Studien und Methoden erfassten Mediennutzungsvariablen hinweg kann aber von recht konsistenten Befunden gesprochen werden. 
und Videospiele, Mädchen lediglich 16 Minuten. Die KIM-Studie 2008 berichtet leider keine nach Jungen und Mädchen aufgeschlüsselten Computerspielzeiten ${ }^{10}$, die Vorgängerstudie KIM 2006 (Feierabend \& Rathgeb, 2007) kommt für Jungen zu ähnlichen Computerspielzeiten (48 Minuten) wie die Studie des KFN, weist allerdings für Mädchen mit 33 Minuten einen deutlich höheren Wert aus als die KFN-Studie. Dennoch ergibt sich auch hier ein deutlicher Geschlechterunterschied. Bezüglich des Fernsehens zeigen sich zwischen den Geschlechtern kaum nennenswerte Unterschiede. Noch am größten erscheint der Geschlechterunterschied in den Daten der KFN-Grundschulbefragung 2005, nach der Jungen an einem Werktag 102 Minuten Fernsehen, Video und DVD schauten und Mädchen 80 Minuten (Mößle et al., 2007). Die KIM-Studie 2008 berichtet dagegen keine Geschlechterunterschiede und auch die verhaltensbasierten Daten der Arbeitsgemeinschaft Fernsehforschung (AGF), in deren Auftrag die Gesellschaft für Konsumforschung (GfK) Fernsehnutzungsdaten erhebt, zeigt für Fernsehzeiten im Alter zwischen 3 und 13 Jahren keinen deutlichen Geschlechterunterschied. Jungen sahen im Jahr 2009 mit 87 Minuten lediglich 3 Minuten länger täglich fern als Mädchen (84 Minuten) (Feierabend \& Klingler, 2010, S. 184).

Neben der Frage, inwieweit das Geschlecht Medienvorlieben und Mediennutzungszeiten beeinflusst, wird immer wieder auf die Bedeutung des elterlichen Bildungshintergrundes für die Rolle verschiedener Medien im Alltag von Kindern hingewiesen (vgl. beispielsweise Kleimann, Mößle, Rehbein \& Pfeiffer, 2006). So kommt die KFN-Grundschulbefragung $2005 \mathrm{zu}$ dem Befund, dass Kinder aus formal hoch gebildeten Elternhäusern ${ }^{11}$ an Schultagen 58 Minuten Fernsehen, Videos und DVDs nutzen, während Kinder aus formal gering gebildeten Elternhäusern 134 Minuten werktäglich mit diesen Medien verbringen (Mößle et al., 2007, S. 61). Bei den Computer- und Videospielzeiten zeigen sich entsprechende Unterschiede. Während Kinder mit hohem formalem Bildungs-

10 Ein deutlicher Geschlechterunterschied in der Nutzungszeit lässt sich jedoch auch hier ableiten: So gab es bei den Jungen doppelt so viele tägliche Computer- bzw. Konsolenspieler (25\%) als bei den Mädchen (11\%) (Feierabend \& Rathgeb, 2009b).

11 Die elterliche Bildung wurde im Rahmen dieser Studie nicht von den Kindern selbst eingeschätzt, sondern durch geschulte Interviewer bei den Klassenlehrerinnen und -lehrern der Kinder erfragt. Dieses Vorgehen erzeugte allerdings eine größere Anzahl von Missings, da die Lehrkräfte sich oftmals nicht zutrauten, zu den Eltern aller Schülerinnen und Schülern verlässliche Angaben zu machen (Baier, Pfeiffer, Windzio \& Rabold, 2006). Als Kinder aus Elternhäusern mit hoher formaler Bildung galten dabei Befragte, von denen mindestens ein Elternteil ein Abitur oder einen Studienabschluss hatte, während Elternhäusern dann eine geringe formale Bildung zugeordnet wurde, wenn beide Eltern höchstens einen Hauptschulabschluss hatten (Mößle et al., 2007, S. 52). 
hintergrund angeben, schultäglich 19 Minuten zu spielen, liegen die Computerund Videospielzeiten bei Kindern niedrig gebildeter Eltern bei 41 Minuten (ebenda).

\subsubsection{Mediengerätebesitz}

Der große Stellenwert des Fernsehens unter Kindern zeigt sich auch in der Ausstattung mit eigenen Fernsehgeräten. Die Daten der KFN-Grundschulbefragung 2005 zeigen für im Schnitt zehnjährige Grundschülerinnen und Grundschüler der vierten Klasse einen eigenen Fernseher bei rund 36 Prozent der Kinder (Mößle et al., 2007) und der Durchschnitt der im Rahmen der KIM-Studie 2008 untersuchten sechs- bis dreizehnjährigen Kinder verfügte $2008 \mathrm{zu} 42$ Prozent über einen eigenen Fernseher im Zimmer (Feierabend \& Rathgeb, 2009b). Der mit acht Prozentpunkten doch beträchtliche Unterschied zwischen KFN- und KIMDaten ist mit einiger Sicherheit zum großen Teil auf die unterschiedlichen Befragungszeitpunkte zurückzuführen ${ }^{12}$. Trotz ähnlicher Fernsehnutzungszeiten von Jungen und Mädchen zeigen sich zwischen den Geschlechtern recht deutliche Unterschiede in der Ausstattung der Kinderzimmer mit eigenen Fernsehgeräten. Die KFN-Grundschulbefragung 2005 ermittelte hier eine Differenz von rund elf Prozent (Mädchen: 31 Prozent; Jungen: 42 Prozent), der Ausstattungsunterschied lag laut KIM 2008 bei nur drei Prozent (Mädchen: 40 \%; Jungen: $43 \%$ ). Bezüglich des Fernsehbesitzes im Kinderzimmer zeigt die KFN-

12 Dabei spiegeln die etwas aktuelleren Daten der KIM-Studie 2008 im Vergleich mit den 2005 erhobenen KFN-Daten einen Trend wieder, der sich auch in einem Vergleich der unterschiedlichen KIM-Studien nachzeichnen lässt: Die KIM 2000 ermittelte einen Fernsehgerätebesitz von Kindern zwischen sechs und dreizehn Jahren von 34 Prozent (Feierabend \& Klingler, 2000). Drei Jahre später wies die KIM 2003 eine Besitzquote von 38 Prozent aus (Feierabend \& Klingler, 2003). Die KIM 2005 kam auf einen Fernsehgerätebesitz von 42 Prozent (Feierabend \& Rathgeb, 2006), die KIM 2006 schließlich auf 44 Prozent eigener Fernseher im Kinderzimmer (Feierabend \& Rathgeb, 2006). Damit liegt in der KIM 2008 der Fernsehgerätebesitz erstmals leicht unter dem Niveau der Vorgängerstudie. Der Unterschied zwischen KIM 2005 (Fernsehbesitzquote: 42\%) und KFN-Viertklässlerbefragung 2005 (36\%) ist höchstwahrscheinlich mit den unterschiedlichen Stichproben zu erklären. So beziehen sich die Auswertungen der KFNSchülerbefragung 2005 allein auf westdeutsche Gebiete, die in den ersten Jahren nach der Wiedervereinigung in der Geräteausstattung der Kinder deutlich hinter den ostdeutschen Bundesländern lagen (Feierabend \& Klingler, 2001). In einer Sonderauswertung der KFN-Viertklässlerbefragung 2005, die parallel zur Hauptuntersuchung auch für das Bundesland Thüringen durchgeführt wurde (Baier et al., 2006) zeigte sich mit einer Fernsehausstattungsquote der Kinder von 50 Prozent eine deutliche Abweichung nach oben gegenüber den meisten westdeutschen Kindern (Kleimann \& Mößle, 2006). 
Grundschulbefragung 2005 beträchtliche Differenzen zwischen Kindern aus formal hoch und formal niedrig gebildeten Elternhäusern: Besitzen Kinder hoch gebildeter Eltern zu lediglich 16 Prozent einen eigenen Fernseher, nennen Kinder formal niedrig gebildeter Eltern zu 57,3 Prozent einen Fernseher ihr Eigen (Mößle et al., 2007, S. 55).

Bezüglich des Besitzes eigener (stationärer) Spielkonsolen zeigte die KFNGrundschulbefragung 2005, dass unter 10-jährigen Viertklässlern rund 27 Prozent der Schülerinnen und Schüler über ein solches Gerät verfügten (Mößle et al., 2007), in der KIM 2008 zeigte sich eine Besitzquote stationärer Spielkonsolen unter sechs- bis dreizehnjährigen Kindern von 25 Prozent (Feierabend \& Rathgeb, 2009b). Im Grundschulalter steigt auch die Ausstattung mit Computern deutlich an. So kommt die KIM 2008 für sechs- bis dreizehnjährige Kinder auf eine Ausstattungsquote mit eigenen Computern von 15 Prozent (ebenda), die KFN-Grundschulbefragung 2005 zeigt für rund 10-jährige Viertklässlerinnen und Viertklässler eine Besitzquote von 36 Prozent $^{13}$ (Mößle et al., 2007). Die deutlich größere Präferenz von Jungen für Computer- und Videospiele macht sich deutlich in der Geräteausstattung der Kinderzimmer bemerkbar. Bei der KFN-Grundschulbefragung 2005 gaben 41 Prozent der Jungen an, einen eigenen Computer im Zimmer zu besitzen, 38 Prozent berichteten von einer eigenen stationären Spielkonsole. Dagegen besaßen lediglich 32 Prozent der Mädchen einen eigenen Computer und nur 16 Prozent eine eigene Spielkonsole (ebenda). Auch die KIM-Studie 2008 kommt mit einer Differenz von 10 Prozent zu einem großen Geschlechterunterschied beim Besitz stationärer Spielkonsolen (Mädchen: $20 \%$; Jungen: $30 \%{ }^{14}$ und zu einer mit sechs Prozent ebenfalls deutlichen Differenz beim Besitz eines eigenen Computers (Mädchen: $12 \%$; Jungen: 18 \%) (Feierabend \& Rathgeb, 2009b).

Beim Besitz von Spielkonsolen und Computern sind - ähnlich wie beim Fernsehbesitz - deutliche Unterschiede zwischen den Bildungsschichten festzustellen. Mößle et al. berichten für Kinder aus hoch gebildeten Elternhäusern eine Besitzquote eigener Spielkonsolen von 11,3 Prozent der Befragten, bei Kindern aus niedrig gebildeten Elternhäusern lag die Besitzquote bei 42,7 Prozent (Mößle et

13 Die doch deutlichen Unterschiede im Ausstattungsniveau der Kinder mit eigenen Computern zwischen der KFN-Befragung und der KIM-Studie sind mutmaßlich mit der größeren Zahl jüngerer Kinder zu erklären, die in der Stichprobe der KIM berücksichtigt werden. Wie die altersspezifischen Analysen der KIM 2002 zeigen, steigt sie Computerausstattung von Kindern aber gerade innerhalb der ersten Grundschuljahre deutlich an.

14 Allerdings haben Mädchen im Vergleich zwischen KIM-2006 und KIM-2008 beim Konsolenbesitz stark aufgeholt. So lag die Geschlechterdifferenz 2006 noch bei 18 Prozentpunkten (Mädchen 13 \%, Jungen $31 \%$ ) (Feierabend \& Rathgeb, 2007). 
al., 2007, S. 55). Beim PC-Besitz fallen die Unterschiede längst nicht so deutlich aus. 32,6 Prozent Kinder hoch gebildeter Eltern hatten 2005 einen eigenen Computer im Zimmer, Kinder gering gebildeter Eltern besaßen zu 42,3 Prozent einen eigenen Computer (ebenda).

\subsubsection{Medieninhalte}

Hinsichtlich der Vorlieben der Kinder bei den Medieninhalten geben sowohl die Daten aus dem AGF/GFK-Fernsehpanel wie auch die Befragungsdaten des Medienpädagogischen Forschungsverbundes Südwest und des Kriminologischen Forschungsinstituts Niedersachsen Aufschluss. Dabei soll im Folgenden auf Fernseh- und Computer(-spiel-)inhalte eingegangen werden.

\subsubsection{Fernsehinhalte}

Unter allen Kindern des AGF/GFK-Fernsehpanels (3 - 13 Jahre) war im Jahr 2009 Super-RTL mit einem Marktanteil von 21,3 Prozent der am meisten genutzte Sender (Feierabend \& Klingler, 2010, S. 190). Am beliebtesten war Super-RTL bei Sechs- bis Siebenjährigen, bei älteren Kindern nimmt die Wichtigkeit dieses Senders proportional zum Lebensalter ab, unter 12- bis 13Jährigen stand er im Jahr 2009 mit einem Anteil von 11,2 Prozent an der Gesamtfernsehzeit dieser Altersgruppe nicht mehr an erster Stelle, sondern belegte hinter Pro7 (15,5\%) und RTL (13,0 \%) den dritten Rang (ebenda). Der Kinderkanal erreichte mit einem Gesamtmarktanteil von 15,5 Prozent unter den Drei- bis Dreizehnjährigen den zweiten Rang bei den Marktanteilen, aber auch hier gilt, dass seine Beliebtheit mit steigendem Alter der Kinder deutlich sinkt (ebenda).

Bereits unter Kindern lassen sich Geschlechterunterschiede in der Beliebtheit bestimmter Fernsehinhalte feststellen. Am beliebtesten sind unter beiden Geschlechtern Zeichentrickformate, allerdings bevorzugen Jungen Zeichentrickserien noch einmal deutlich stärker als Mädchen (in $61 \%$ der Zeit, die Jungen Fernsehserien schauen, nutzen sie Zeichentrickserien, Mädchen in 47\%) (S. 193). Insgesamt stellen fiktionale Formate (Spannung, Komödie, Unterhaltung/Drama, Animation) unter Jungen (39\%) wie Mädchen (38\%) das am häufigsten genutzte Format dar, während Informations- (Marktanteil: $22 \%$ ) und Unterhaltungsprogramme (14\%) deutlich weniger wichtig sind (ebenda). Im Rahmen der KIM 2008 wurden Kinder konkret nach ihren Lieblingssendungen 
im Fernsehen gefragt. Die unter Kindern beliebteste Sendung mit neun Prozent aller Nennungen war im Jahr 2008 demnach Spongebob, eine Zeichentrickserie die auf Super-RTL ausgestrahlt wird, und die besonders von Kindern zwischen sechs und sieben Jahren häufig genannt wurde (19\%). Die zweitbeliebteste Fernsehsendung war 2008 die auf RTL ausgestrahlte Daily Soap Gute Zeiten Schlechte Zeiten (6\%), die besonders unter Mädchen sehr beliebt ist (11\%).

\subsubsection{Nicht altersgemäße Filme und Fernsehsendungen}

Im Rahmen der KFN-Grundschulbefragung 2005 wurden die Kinder neben ihren allgemeinen Fernsehsenderpräferenzen auch nach ihren Erfahrungen mit Filmen und Fernsehsendungen befragt, die erst für Jugendliche ab 16 Jahren beziehungsweise Erwachsene freigegeben sind. 18 Prozent der Mädchen und 34,4 Prozent der Jungen gaben an, in der letzten Woche einen solchen Film beziehungsweise eine solche Sendung gesehen zu haben (Mößle et al., 2007, S. 70). Gruppenspezifische Auswertungen zeigten, dass Kinder aus formal niedrig gebildeten Elternhäusern rund dreimal so häufig nicht altersgemäße Inhalte nutzten wie Kinder aus formal hoch gebildeten Elternhäusern (ebenda), und dass Kinder mit eigenem Fernsehgerät im Zimmer rund doppelt so häufig Filme oder Fernsehsendungen ,ab 16/18“ schauten, als Kinder ohne ein eigenes Fernsehgerät im Zimmer (S. 65).

\subsubsection{Computerspielinhalte}

Im Bereich der Computer- und Videospiele können Kinder aus einem äußerst breiten inhaltlichen Angebot auswählen. So berichtete die in Deutschland für die Alterseinstufung aller auf Trägermedien veröffentlichten Computer- und Videospiele beauftragte Unterhaltungssoftware-Selbstkontrolle (USK) von jährlich 2607 (Jahr 2006) bis 3100 (Jahr 2009) Prüfverfahren für unterschiedliche Spieletitel $^{15}$ (Unterhaltungssoftware Selbstkontrolle (USK), 2007, 2010). 65,2 Prozent dieser Titel bekamen im Jahr 2009 die Altersfreigabe „ohne Altersbeschränkung“ beziehungsweise „,ab 6 Jahren“, so dass knapp zwei Drittel aller

15 Dabei ist allerdings zu berücksichtigen, dass es sich dabei nicht immer um unterschiedliche Spiele handelt. Viele Spieltitel werden parallel für unterschiedliche Plattformen (PC, Playstation 2 oder 3, Nintendo Wii, etc...) publiziert und müssen für jede Plattform einzeln bei der Unterhaltungssoftware Selbstkontrolle (USK) eingereicht werden. 
eingereichten Spiele zumindest theoretisch für Kinder im Grundschulalter erhältlich waren (Unterhaltungssoftware Selbstkontrolle (USK), 2010). Im Durchschnitt besaßen Kinder zwischen sechs und dreizehn Jahren im Jahr 200814 eigene Computer- oder Videospiele, Jungen verfügten über deutlich mehr Spiele (17) als Mädchen (10) (Feierabend \& Rathgeb, 2009b, S. 29). Auch bei den favorisierten Spieltiteln und Spielgenres zeigen sich deutliche geschlechtsspezifische Unterschiede: Jungen spielen sehr viel häufiger als Mädchen Sport-, Adventure- und verschiedene Actionspiele, Mädchen nutzen häufiger als Jungen Alltagssimulationsspiele, Fun- oder Partyspiele sowie Lernspiele (Feierabend \& Rathgeb, 2009b, S. 30 - 31; Mößle, 2009, S. 18 - 21).

\subsubsection{Nicht altersgemäße Computer- und Videospiele}

Auch wenn ein Großteil der unter Kindern beliebtesten Titel höchstens Alterseinstufungen „ab 6 Jahren“ erhalten hat, zeige die KIM 2008, dass 30 Prozent der sechs- bis dreizehnjährigen Kinder bereits Spiele gespielt zu haben, für die sie noch nicht alt genug sind (Feierabend \& Rathgeb, 2009b, S. 31), wobei Jungen deutlich häufiger betroffen waren (35\%) als Mädchen (23\%). Auch im Rahmen der KFN-Grundschulbefragung 2005 gaben 33,5 Prozent aller befragten Viertklässlerinnen und Viertklässler an, bereits ein Spiel mit einer Freigabe ,ab 16 Jahren“ gespielt zu haben (Jungen: 50,1\%; Mädchen: 16,7\%), 18 Prozent eines mit einer Freigabe „ab 18 Jahren“ (Jungen: 28,9\%; Mädchen: 7,2\%) (Mößle, 2009, S. 21). Mößle et al. (2007) baten die befragten Kinder, im Rahmen der KFN-Grundschulbefragung 2005 anzugeben, welche drei Spiele sie derzeit am meisten spielen. Rund ein Fünftel der Jungen, aber lediglich 3 Prozent der Mädchen gaben dabei mindestens ein Spiel mit einer Freigabe „ab 16 oder 18 Jahren“ an, so dass insgesamt 12,2 Prozent aller Kinder ein solches Spiel spielten (ebenda). Als am häufigsten genutztes Spiel mit einer Altersfreigabe „ab 16 Jahren“ nennen Mößle et al. (2007, S. 62) in diesem Kontext das Spiel Grand Theft Auto (GTA), ein Third-Person-Action-Game, welches im Jahr 2005 von 5,7 Prozent aller befragten Kinder gespielt wurde (Jungen: 10,0\%; Mädchen: 1,4\%).

Das Spielen nicht altersgemäßer Computer- und Videospiele ist mithin insbesondere unter Jungen zu finden. Zudem zeigen Mößle et al. (2007, S. 70), dass Kinder aus Elternhäusern mit niedriger formaler Bildung rund dreimal so häufig regelmäßig nicht altersgemäße Spiele „ab 16 Jahren“ spielen wie Kinder mit formal hoch gebildeten Eltern. Darüber hinaus weisen die Autoren auf große Unterschiede zwischen Kindern mit beziehungsweise ohne eigene Spielkonsole im Zimmer hin. So legen sie beispielsweise dar, dass Kinder, deren Spielkonsole 
im eigenen Zimmer stand, zu 24,4 Prozent zum Befragungszeitpunkt ein Videospiel mit einer Freigabe „ab 16 Jahren“ spielten, während Kinder, deren Spielkonsole anderswo im Haus aufgestellt war, dies nur zu 10,7 Prozent angaben (2007, S. 67).

\subsection{Jugendliche und Medien}

\subsubsection{Mediennutzungszeiten}

Im Leben deutscher Jugendlicher gewinnen Medien im Vergleich zu jüngeren Minderjährigen abermals an Bedeutung, es verschieben sich allerdings deutlich die Schwerpunkte zwischen den Medien. Im Rahmen der Studie Jugend und Medien (JIM) 2009 erfasste der Medienpädagogische Forschungsverbund Südwest in ähnlicher Weise wie in den KIM-Studien die Freizeit und Medieninteressen von 1.200 deutschland-repräsentativ telefonisch befragten 12- 19jährigen Jugendlichen (Feierabend \& Rathgeb, 2009a). Der größte Unterschied zum Freizeitverhalten von Kindern zeigt sich in der breiten täglichen Verwendung der Medien durch Jugendliche. Vier von fünf Jugendlichen gaben im Rahmen der JIM-2009-Studie an, täglich ein Handy zu nutzen (79\%), rund zwei Drittel nutzten täglich das Internet (65\%) oder den Fernseher (63\%) (Feierabend \& Rathgeb, 2009a, S. 16 - 17). Die größten Unterschiede zwischen weiblichen und männlichen Jugendlichen offenbarten sich bei der Nutzung von Büchern sowie von nicht internetgestützten Computer- und Konsolenspielen. So lasen Mädchen sehr viel häufiger (52\% täglich/mehrmals wöchentlich) als Jungen (29\%). Offline-Computer- und Konsolenspiele wurden von Jungen zu 53 Prozent mindestens mehrmals wöchentlich genutzt, von Mädchen lediglich zu 17 Prozent. Recht deutliche Geschlechterunterschiede zeigen sich auch bei der Handynutzung und der DVD- beziehungsweise Videorezeption. Mädchen nutzten häufiger das Handy (täglich/mehrmals pro Woche: $92 \%$; Jungen: 84 \%), Jungen schauten mehr Videos und DVDs (täglich/mehrmals pro Woche: $33 \%$; Mädchen: $25 \%$ ) (ebenda).

Feierabend und Rathgeb untersuchten im Rahmen der JIM-Studie 2009 auch die subjektive Relevanz der Medien für die Jugendlichen (S. 19 - 21). Musik hören und Internetnutzung waren für knapp 90 Prozent der Befragten die wichtigste Mediennutzungsform, es folgten die Handynutzung (72\%), das Fernsehen $(69 \%)$ und das Lesen von Büchern (53\%). Damit zeigt sich wie bei der Nutzungsfrequenz, dass unter den Jugendlichen die Verwendung des Internet inzwischen eine wichtigere Rolle spielt als das Fernsehen. Die deutlichsten 
Geschlechterunterschiede von mehr als 20 Prozentpunkten zeigen sich bezüglich der Wichtigkeit von Büchern und Computerspielen. Mädchen gaben zu 64 Prozent an, das Lesen von Büchern sehr wichtig oder wichtig zu finden, damit nahm das Lesen von Büchern den fünften Platz in der Wichtigkeit der Medientätigkeiten ein. Unter den Jungen bezeichneten hingegen nur 42 Prozent Bücherlesen als sehr wichtig oder wichtig, damit lag diese Tätigkeit auf dem achten Platz der wichtigen Medien. Fast spiegelbildlich stellt sich diese Geschlechterdifferenz bei der Nutzung von Computerspielen dar. Für 65 Prozent der Jungen war Computerspielen wichtig oder sehr wichtig, damit lag es auf dem vierten Platz der Wichtigkeitsrangfolge. Bei Mädchen lag dieser Wert bei lediglich 24 Prozent, damit lag Computer spielen auf dem achten Platz (ebenda).

Die Relevanz der Medien Internet, Fernsehen und Computer(spiele) findet sich auch in den Daten der KFN-Schülerbefragung 2007/2008 wieder, in der 44.601 jugendliche Neuntklässlerinnen und Neuntklässler in 61 zufällig ausgewählten Städten und Landkreis befragt wurden, so dass von einem deutschland-repräsentativen Sample 15-jähriger Jugendlicher ausgegangen werden kann (Baier, Pfeiffer, Simonson \& Rabold, 2009). Im Rahmen dieser Befragung ergaben sich tägliche Fernsehnutzungszeiten (inkl. DVD und Video) von 201 Minuten für Jungen sowie 213 Minuten für Mädchen, eine tägliche Nutzungszeit von Computer- und Videospielen (Online und Offline) von knapp einer Stunde (56 Minuten) für Mädchen und 141 Minuten für Jungen ${ }^{16}$ sowie etwas mehr als 100 Minuten Internetchatnutzung für beide Geschlechter (Mädchen: 113 Minuten, Jungen: 103 Minuten) ${ }^{17}$ (Rehbein, Kleimann \& Mößle, 2009b, S. 15 - 16).

16 Der Vergleich von JIM-2009-Daten und denen der KFN-Schülerbefragung 2007/2008 zeigt, dass die Daten der JIM recht deutlich unter denen des KFN liegen. Gaben Jungen im Rahmen der JIM 2009 an, 98 Minuten Video- und Computerspiele zu spielen (online und offline), waren es bei der KFN-Befragung 141 Minuten. Bei den Mädchen dagegen lagen die Daten der JIM 2009 (53 Minuten) nur leicht unter denen des KFN (56 Minuten). Ein Hauptgrund der Differenz beider Studien ist in der Tatsache zu vermuten, dass in die durchschnittlichen Nutzungszeiten der KFN-Befragung die Wochenendnutzung eingeflossen ist, während im Rahmen der JIM 2009 explizit nach werktäglicher Nutzung gefragt wurde. Bereits Mößle et al. (Mößle et al., 2007, S. 81) konnten indes zeigen, dass sich insbesondere die Computerspielzeiten von männlichen Jugendlichen am Wochenende gegenüber der werktäglichen Nutzung um rund 50 Minuten erhöhen.

17 Bezüglich der Internetnutzung kann zwischen JIM 2009 und KFN-Schülerbefragung 2007/2008 kaum verglichen werden, da im Rahmen der JIM-Studie nur die Gesamtinternetzeit (ohne Onlinecomputerspiele) abgefragt wurde. Diese lag mit 146 Minuten bei Jungen und 121 Minuten bei Mädchen wenig überraschend über den Daten der KFNStudie (Feierabend \& Rathgeb, 2009a, S. 32). Im Rahmen der JIM 2009 war Chatten über 
Die in der KFN-Schülerbefragung 2007/2008 erfassten Lesezeiten zeigen im Vergleich zu den Viertklässlern der KFN-Grundschulbefragung, dass dem Lesen in der Freizeit insbesondere bei Jungen mit Erreichen der Adoleszenz weniger Bedeutung zukommt. So lasen männliche Jugendliche lediglich 25 Minuten täglich (unter den männlichen wie weiblichen Grundschülern 2005 waren es noch rund 50 Minuten täglich), weibliche Jugendliche lasen 43 Minuten (Mößle et al., 2007, S. 58; Rehbein et al., 2009a, S. 16). Lesen besitzt mithin weiterhin einen gewissen Stellenwert für Jugendliche, in der täglichen Nutzung nimmt diese Tätigkeit aber mit zunehmendem Alter ab und wird damit gegenüber den zunehmend genutzten elektronischen Medien weniger wichtig. Innerhalb der elektronischen Medien verliert das Fernsehen trotz weiterhin hoher Nutzungszeiten seinen Stellenwert als klares Leitmedium zunehmend an das Internet, welches im Stellenwert unter den Jugendlichen bereits das Fernsehen überholt hat und in der täglichen Nutzungszeit mit dem Fernsehen gleichzieht.

\subsubsection{Mediengerätebesitz}

Der Siegeszug der digitalen Medien lässt sich auch an der Geräteausstattung der Jugendlichen ablesen. Zunächst steigt in der Adoleszenz die Ausstattung mit eigenen Fernsehern im Gegensatz zum Kindesalter noch einmal deutlich an. Die JIM 2009 kommt zu einer Fernsehausstattungsquote im eigenen Zimmer der befragten 12- bis 19-jährigen Jugendlichen von 60 Prozent, die KFNSchülerbefragung 2007/2008 ermittelte für durchschnittlich 15-jährige Jugendliche 69,5 Prozent eigene Fernseher im Zimmer (Feierabend \& Rathgeb, 2009a, S. 7 - 8; Rehbein et al., 2009b, S. 17). Die Geschlechterdifferenzen in der Ausstattung mit Fernsehgeräten fallen laut KFN-Schülerbefragung 2007/2008 mit rund 10 Prozentpunkten (Jungen: 74,3 \%; Mädchen: 64,4 \%) noch recht deutlich aus, in der JIM 2009 mit ergab sich lediglich ein minimaler Besitzvorsprung der männlichen Jugendlichen (Jungen: $61 \%$; Mädchen: 60\%) (ebenda). Die JIM 2009 berichtet für 12- bis 19-jährige Jugendliche eine Besitzquote für stationäre Spielkonsolen von 45 Prozent, fast ebenso die KFN-Schülerbefragung 2007/2008 mit rund 46 Prozent (ebenda). Besonders zeigt sich an der Geräteausstattung mit Computern der Wandel der Medienwelt zwischen Kindheit und Adoleszenz. Da der Computer weit stärker als im Kindesalter für schulische Aufgaben und für die Kommunikation mit Freunden genutzt wird, steigt die Be-

Instant Messenger die am stärksten frequentierte Internet-Funktion, chatten in Chaträumen die vierthäufigste Nutzungsart (Feierabend \& Rathgeb, 2009a, S. 34). 
sitzquote laut KFN-Schülerbefragung 2007/2008 auf rund 70 Prozent, die Daten der JIM 2009 gleichen mit 71 Prozent Besitzquote fast exakt denen des KFN (ebenda). Damit liegt die Ausstattung mit Computern inzwischen leicht über der Fernsehausstattung.

Erwartungsgemäß zeigen sich auch bei den Jugendlichen bezüglich der Mediengeräteausstattung deutliche Geschlechterunterschiede. Am stärksten fallen diese beim Besitz stationärer Spielkonsolen aus. Sowohl die JIM-Studie 2009 wie auch die KFN-Schülerbefragung 2007/2008 berichten hier von deutlichen Unterschieden zwischen 23 und 34 Prozentpunkten Differenz zugunsten der Jungen (JIM: Jungen: 56\%; Mädchen: $33 \%$; KFN: Jungen: 62,5\%; Mädchen: 28,3\%). Beim Computerbesitz zeigt sich ein Unterschied von 17 Prozentpunkten im Rahmen der KFN-Schülerbefragung 2007/2008 (Mädchen: 61,2 \%; Jungen: 78 \%) beziehungsweise von fünf Prozentpunkten im Rahmen der JIM-Studie 2009 (Mädchen: $72 \%$; Jungen: 77 \%) (ebenda).

\subsubsection{Medieninhalte}

\subsubsection{Fernsehinhalte}

Im Rahmen der JIM 2009 wurden Jugendliche zumindest auf Senderebene zu ihren inhaltlichen Fernsehpräferenzen befragt (Feierabend \& Rathgeb, 2009a, S. 27 - 28). Dabei ergab sich wie bereits in den Vorläuferstudien eindeutig, dass Jugendliche private Fernsehprogramme präferieren. Beliebtester Fernsehsender unter den Jugendlichen war mit weitem Abstand Pro7. 45 Prozent aller Befragten nannten ihn im Rahmen der JIM 2008 als ihren Lieblingssender. Es folgten RTL (13\%), Sat.1 (6\%) und MTV (5\%). Auch bei den Jugendlichen zeigten sich hinsichtlich der Fernsehsenderpräferenz deutliche Geschlechterunterschiede, wobei der bei beiden Geschlechtern auf dem ersten Platz liegende Sender Pro7 bei Jungen (50\%) noch etwas beliebter war als bei Mädchen (40\%). Dagegen nannten Mädchen zu 18 Prozent RTL als Lieblingssender, Jungen zu 9 Prozent (ebenda).

Wie bei den Kindern zeigt sich auch bei Jugendlichen, dass die Zahlen für Fernsehgerätebesitz und tägliche Fernsehnutzung, für Videospielkonsolenbesitz und Video- und Computerspielzeiten dann am höchsten sind, wenn die Jugendlichen aus Elternhäusern mit niedrigem formalen Bildungshintergrund stammen und/oder derzeit die Hauptschule besuchen (Mößle et al., 2007). Lediglich hinsichtlich der Ausstattung mit Computern, die in allen weiterführenden Schulformen inzwischen auch für viele schulische Aufgaben genutzt werden, ergeben 
sich kaum Ausstattungsunterschiede zwischen Bildungsschichten und Schulformen. Auch hinsichtlich der Internetnutzungszeiten (nicht mitgerechnet internetbasierte Computerspiele) unterscheiden sich Schülerinnen und Schüler verschiedener Schulformen und verschiedener Bildungshintergründe inzwischen kaum mehr, während das Internet vor einigen Jahren noch als Medium höher gebildeter Jugendlicher galt (Feierabend \& Rathgeb, 2009a; Mößle et al., 2007).

2.3.3.2 Nicht altersgemäße Filme und Fernsehsendungen

Die Nutzung nicht altersgemäßer Film- und Fernsehinhalte ist unter Jugendlichen weit verbreitet. Die im Rahmen der KFN-Neuntklässlerbefragung $2005^{18}$ untersuchten 15-jährigen Jugendlichen gaben zu 56,6 Prozent an, mindestens mehrmals im Monat Actionfilme zu schauen, die ab 16 Jahren freigegeben sind. 40,2 Prozent sagten, mehrmals monatlich oder häufiger Filme mit einer Freigabe von 18 Jahren anzuschauen (Mößle et al., 2007, S. 85). Die Nutzung der in dieser Abfrage noch nicht berücksichtigten Horrorfilme wurde entsprechend einzeln abgefragt. 35,6 Prozent schauten mindestens mehrmals im Monat Horrorfilme „ab 16“, 25,3 Prozent nutzten Horrorfilme „ab 18“. Hinzu kamen noch Sex- oder Pornofilme, die von 18,2 Prozent mehrmals im Monat oder häufiger genutzt wurden (ebenda). Jungen schauten alle genannten Filmgenres deutlich häufiger als Mädchen, so dass zumindest für männliche Jugendliche festgestellt werden kann, dass insbesondere die Nutzung gewalthaltiger Actionfilme einen festen Bestandteil im Medienalltag innehat. So nutzten knapp drei Viertel der Jungen $(73,2)$ mindestens mehrmals monatlich Filme „ab 16“, 57,1 Prozent schauten entsprechend häufig Filme ,ab 18“ (S. 86). Horrorfilme „ab 16/18“ sowie Sexund Pornofilme wurden von einem runden Drittel der Jungen in entsprechender Frequenz genutzt (ebenda). Spielte die Rezeption erotischer beziehungsweise pornografischer Formate für Mädchen kaum eine Rolle $(1,8 \%)$, wurden die anderen Gewaltfilmformate von weiblichen Befragten zu knapp 20 bis rund 40 Prozent mit einiger Regelmäßigkeit genutzt (Horrorfilme ,ab 18: 18,3\%; Actionfilme ,ab 18“: 23,2\%; Horrorfilme ,ab 16“: 31,7\%; Actionfilme ,ab 16“: $40,1 \%)$. Im Jugendalter ist also - ebenso wie bei Kindern - nicht altersgemäße Filmnutzung maßgeblich durch das Geschlecht vermittelt. Zudem zeigen sich wenn auch nicht so deutlich wie bei den Viertklässlern - Effekte des elterlichen

18 Die KFN-Neuntklässlerbefragung 2005 war die Vorläuferstudie der KFNSchülerbefragung 2007/2008. Im Rahmen der KFN-Neuntklässlerbefragung 2005 wurden 14.301 jugendliche Neuntklässler in elf westdeutschen Städten und Landkreisen befragt (Mößle et al., 2007, S. 113 - 126). Die Befragung war für die Population die Neuntklässler/innen der beteiligten Städte und Landkreise repräsentativ. 
Bildungshintergrundes (S. 90). So gaben Jugendliche mit niedrigem formalen Bildungshintergrund im Elternhaus zu 18 Prozent an, täglich oder mehrmals pro Woche Gewaltfilme ,ab 18“ anzuschauen, während dies unter Jugendlichen aus formal hoch gebildeten Elternhäusern lediglich 10,9 Prozent taten (ebenda).

\subsubsection{Computer- und Videospielinhalte}

Auch im Jugendalter unterscheiden sich Genrepräferenzen für Computer- oder Videospiele sehr deutlich zwischen Jungen und Mädchen. Weibliche Jugendliche bevorzugen in erster Linie Alltagssimulationen, Geschicklichkeits- und Gelegenheitsspiele sowie Karaokespiele, männliche Jugendliche spielen am häufigsten Shooter- und Actionspiele sowie Sport- und Rennspiele (Feierabend \& Rathgeb, 2009a, S. 42; Mößle, 2009, S. 18 - 21) ${ }^{19}$. Im Vergleich zur Gruppe der Kinder zeigt sich bei Jugendlichen ein deutlich diversifiziertes Spektrum an Nutzungskonstellationen: Insgesamt dominiert unter Jugendlichen die Nutzung von Multiplayer-Onlinespielen, was vor allem auf die starke Präferenz von Jungen für solche Formate zurückzuführen ist. Erst danach folgen in ungefähr gleicher Nutzungsfrequenz das alleinige Spielen an einer Spielkonsole, an einem PC oder eines Online-Spiels ${ }^{20}$ (Feierabend \& Rathgeb, 2009a, S. 40). Das gemeinsame Spielen eines Offlinespiels an einem PC oder einer Spielkonsole verliert gegenüber diesen Nutzungsformen an Bedeutung (ebenda). Auch bei den Ergebnissen der verschiedenen Befragungen von Jugendlichen $\mathrm{zu}$ ihren Computerspielvorlieben zeigen sich deutliche Übereinstimmungen zwischen KFN-Schülerbefragung 2005 und JIM 2009. Beim Vergleich der JIM 2009 mit der vier Jahre vorher durchgeführten KFN-Schülerbefragung 2005 zeigt sich bei relativer Stabilität der Spielepräferenzen der Jugendlichen der große Beliebtheitssprung des Spiels Word of Warcraft. Kam dieser Titel im Jahr 2005 knapp nicht unter die zehn beliebtesten Spieltitel, wurde er im Rahmen der JIM 2009 als viertbeliebtestes Spiel unter den Jungen gemessen (Feierabend \& Rathgeb,

19 Bei der Analyse der beliebtesten Spielgenres werden neben den Daten der JIM im Folgenden die Daten der KFN-Neuntklässlerbefragung 2005 berichtet, da hier im Vergleich zur neueren KFN-Schülerbefragung 2007/2008 stärker ausdifferenzierte Analysen bezüglich der Spielvorliebe der Jugendlichen vorliegen und die Daten daher besser mit denen der JIM-Studie vergleichbar sind. Die Trends der KFN-Neuntklässlerbefragung 2005 werden, soweit vergleichbar, durch die KFN-Schülerbefragung 2007/2008 weitgehend bestätigt. Erste Ergebnisse der KFN-Schülerbefragung 2007/2008 zu Genrevorlieben von Jugendlichen finden sich bei Rehbein, Kleimann und Mößle (2009b, S. 17 18).

20 Vorzugsweise sind hier wohl browserbasierte Flashgames gemeint. 
2009a, S. 42). Außerdem zeigte sich Rahmen der JIM 2009, dass die neue Kategorie der Karaokespiele, die vier Jahre vorher noch keine besonders bedeutende Rolle spielte, unter den Mädchen inzwischen sehr beliebt ist. So kam das Spiel Singstar bei den befragten Mädchen auf den zweiten Platz der Beliebtheitsskala (Feierabend \& Rathgeb, 2009a, S. 41).

\subsubsection{Nicht altersgemäße Computer- und Videospiele}

Wie bereits gezeigt wurde, sind die beliebtesten Spielgenres männlicher Jugendlicher Shooter- und Actionspiele. Dabei ist darauf hinzuweisen, dass die am häufigsten gespielten Titel dieser Gattung - Counter Strike und GTA - für ihre verschiedenen Einzeltitel mindestens Altersfreigaben ab 16 Jahren, in Einzel-

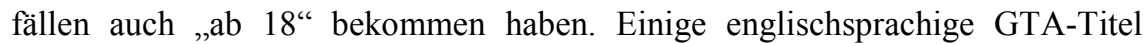
wurden von der Bundesprüfstelle für Jugendgefährdende Medien indiziert (Bundesprüfstelle für jugendgefährdende Medien (BPjM), 2010b). Auch der beliebte Kriegsshooter Call of Duty ist in allen deutschen Versionen der Serie aus-

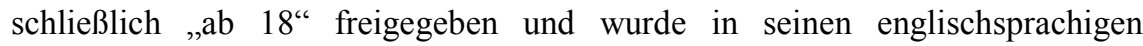
Versionen mehrfach indiziert (ebenda). Insgesamt gaben unter den befragten Jugendlichen der KFN-Neuntklässlerbefragung 2005, die noch nicht 16 Jahre alt waren, 68,2 Prozent an, schon einmal ein Spiel mit einer Altersfreigabe ab 16 Jahren gespielt zu haben. Unter den Jungen hatte fast jeder bereits ein solches Spiel gespielt $(93,7 \%)$, unter den Mädchen nicht ganz die Hälfte der Befragten (46,3\%) (Mößle, Kleimann \& Rehbein, 2008). Auch mit Spielen ,ab 18“ hatte eine Mehrheit der Befragten bereits Erfahrung. 53,5 Prozent aller 2005 befragten Neuntklässler unter 18 Jahren haben nach eigenen Angaben schon einmal ein Spiel ohne Jugendfreigabe (ab 18) gespielt (Mädchen: 26,6 \%; Jungen: 82,1 $\%)$ (ebenda).

Mößle et al. (2007) analysierten detailliert die Angaben der Schülerinnen und Schüler aus dem Jahr 2005, welche drei Spiele derzeit am meisten gespielt wurden. Hier zeigte sich, dass 14,1 Prozent der befragten unter 18-Jährigen zum Befragungszeitpunkt ein Spiel ohne Jugendfreigabe (ab 18) spielten (Mädchen: 2,2 \%; Jungen: 25,9 \%)(ebenda), 4,4 Prozent der Jungen und nur 0,4 Prozent der Mädchen nannten darüber hinaus ein eindeutig indiziertes Spiel (Mößle et al., 2007, S. 88). Im Gegensatz zum Konsum nicht altersgemäßer Filme durch Jugendliche (vgl. S. 41) und zur Nutzung gewalthaltiger Medien durch Kinder (vgl. S. 35 - 36) lässt sich bezüglich der Nutzung nicht altersgemäßer Computerund Videospiele durch Jugendliche kein Zusammenhang mit dem elterlichen Bildungshintergrund feststellen (Mößle et al., 2007, S. 90). Feierabend und 
Rathgeb stellen lediglich eine etwas größere Erfahrung von Hauptschülerinnen und Hauptschülern mit solchen Spielen fest (72\%) als bei Schülerinnen und Schülern auf Gymnasien (62\%) und Realschulen (63\%) (Feierabend \& Rathgeb, 2009a, S. 43). Offensichtlich betrifft das Problem der Nutzung nicht altersgemäßer Computer- und Videospiele jugendliche Jungen aus allen gesellschaftlichen Schichten in ähnlicher Weise. 